\title{
Efficacy of MCl-186, a free-radical scavenger and antioxidant, for resuscitation of nonbeating donor hearts
}

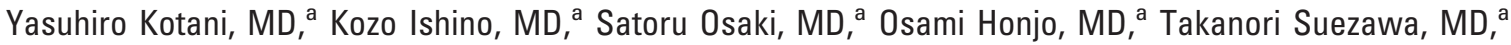 \\ Kazushige Kanki, MD, ${ }^{\text {a }}$ Chikao Yutani, MD, ${ }^{b}$ and Shunji Sano, MD ${ }^{a}$
}

From the Department of Cardiovascula Surgery, Okayama University Graduate School of Medicine and Dentistry, ${ }^{\mathrm{a}}$ and the Department of Life Science, Faculty of Science, Okayama University of Science, ${ }^{\mathrm{b}}$ Okayama, Japan.

Supported by grants from Grant-in-Aid for Scientific Research (No. 16591397-00) from the Japan Society for Promotion of Science. MCI-186 was a gift from Mitsubishi Pharma Corporation, Osaka, Japan.

Received for publication Nov 6, 2006; revisions received Jan 24, 2007; accepted for publication Jan 30, 2007.

Address for reprints: Kozo Ishino, MD, Department of Cardiovascular Surgery, Okayama University Graduate School of Medicine and Dentistry, 2-5-1 Shikata, Okayama City, Okayama, 700-8558, Japan (E-mail: ishino@tb3.so-net.ne.jp).

J Thorac Cardiovasc Surg 2007;133:1626-32 $0022-5223 / \$ 32.00$

Copyright (๑) 2007 by The American Association for Thoracic Surgery

doi:10.1016/j.jtcvs.2007.01.068
Objective: Oxygen-derived free radicals are responsible in part for reperfusion injury in globally ischemic myocardium. In this study, the efficacy for resuscitation of nonbeating donor hearts of MCI-186, a free-radical scavenger and antioxidant, was investigated in a pig transplantation model.

Methods: Cardiac arrest was induced by asphyxiation. After 30 minutes of global ischemia, the hearts were excised and immediately reperfused from the aortic root with normoxemic blood cardioplegia $\left(\mathrm{PO}_{2} 100 \mathrm{~mm} \mathrm{Hg}\right)$ for 20 minutes, followed by perfusion with hyperoxemic blood $\left(\mathrm{Po}_{2} 300 \mathrm{~mm} \mathrm{Hg}\right)$. MCI-186 $(3 \mathrm{mg} / \mathrm{kg})$ was administered into the aortic root for the first 30 minutes of reperfusion in the treated group $(\mathrm{n}=6)$, and untreated hearts were used as a control group $(\mathrm{n}=6)$. Transplantation was performed with the heart beating.

Results: Posttransplantation recovery of cardiac output, end-systolic pressure-volume ratio, and first derivative of pressure of the left ventricle in the treated group were significantly better than those in the control group. The coronary sinus-aortic root difference in malondialdehyde levels remained low throughout reperfusion in the treated group but abruptly increased after initiation of oxygenated blood perfusion in the control group. The MCI-186-treated hearts showed low degree of edema and well-preserved ultrastructure with normal-appearing organelles, whereas the untreated hearts had marked swelling of mitochondria and scant glycogen granules.

Conclusion: MCI-186 exerts a cardioprotective action at least partly by inhibition of lipid peroxidation. Antioxidant therapy at the initial reperfusion is essential to successful resuscitation of nonbeating hearts by continuous myocardial perfusion.

A $\mathrm{n}$ increasing lack of brain-dead donors and a growing number of patients in need of cardiac transplantation has prompted clinicians to reevaluate the potential use of cardiac allograft from non-heart beating donors (NHBDs). This treatment strategy has not yet been used beyond the laboratory, however, because profound myocardial damage developing during an indeterminate period of warm ischemia is unavoidable. Recent studies in animal models have shown that preservation of NHBD cardiac graft with simple cardioplegic flush and hypothermic storage allows functional recovery only when multiple cardioprotective pretreatments are used. ${ }^{1,2}$ Because any treatment before death in anticipation of possible donation is ethically unacceptable, establishment of legally defensible techniques for resuscitation of cadaver hearts and of specific measures for myocardial protection after death is critical to ensure the feasibility of using such donor hearts for human transplantation.

Investigators in 1960s attempted to restore canine and human cadaver hearts by resumption of coronary perfusion with normothermic oxygenated blood ${ }^{3,4}$ but without consideration of reperfusion injury. When cardiomyocytes are reoxygenated after a prolonged period of ischemia, cytosolic calcium overload and reactivation of 


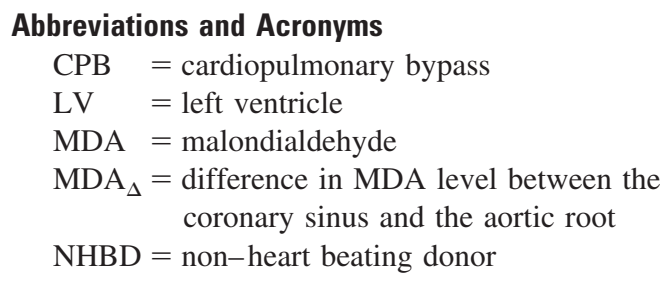

energy production cause deleterious hypercontracture. ${ }^{5}$ Thus the initial reperfusion should maintain cardiac arrest, allowing a brief interval where ionic balance can recover more normally during reoxygenation. To reduce myocardial ischemia before transplantation, we have attempted to resuscitate nonbeating hearts with a continuous myocardial perfusion technique, but without the use of any cardioprotective pretreatments. Recent work from our laboratory demonstrated the importance of the duration of the initial controlled reperfusion with leukocyte-depleted lukewarm blood cardioplegic solution. ${ }^{6}$

There is a wealth of evidence that oxygen free radicals play major roles in ischemia-reperfusion injury. ${ }^{7}$ On reperfusion, molecular oxygen undergoes sequential reduction to form reactive species of oxygen, including superoxide anion and hydroxyl radical. The interaction of oxygen-derived free radicals with lipids and essential proteins in the cell membrane contributes to myocardial damage. ${ }^{8}$ MCI-186 (3-methyl-1-phenyl-2-pyrazolin-5-one) is an interesting compound with unique pharmacologic actions; it can act as a hydroxyl radical scavenger ${ }^{9}$ and has antioxidant effects on both hydroxyl radical-dependent and iron-dependent lipid peroxidation. ${ }^{10,11}$ In Japan, this agent has been used clinically to treat patients with cerebral infarction, ${ }^{12}$ and its neuroprotective effects are indisputable. In this study we investigated the cardioprotective effects of MCI-186 on hearts obtained from asphyxiated NHBDs in a pig transplantation model by administration of this agent during the initial reperfusion.

\section{Materials and Methods \\ Animal Preparation}

All experimental animals were cared for in accordance with institutional guidelines and the "Guide for the Care and Use of Laboratory Animals" (http://www.nap.edu/catalog/5140.html). The experimental protocol was approved by the Okayama University School of Medicine experimental animals committee. Twelve weight-matched pairs of Yorkshire pigs (male or female, mean weight $20 \pm 3 \mathrm{~kg}$ ) were used. Another pig was used for blood donation at each experiment. After premedication with intramuscular injection of $10-\mathrm{mg} / \mathrm{kg}$ ketamine hydrochloride, an ear vein was cannulated, and anesthesia was induced with $50 \mathrm{mg}$ thiamylal sodium and $0.5 \mathrm{mg}$ atropine sulfate and maintained with isoflurane inhalation $(0.5 \%-2.0 \%)$ and $0.2-\mathrm{mg} / \mathrm{kg}$ pancuronium. An endotra-

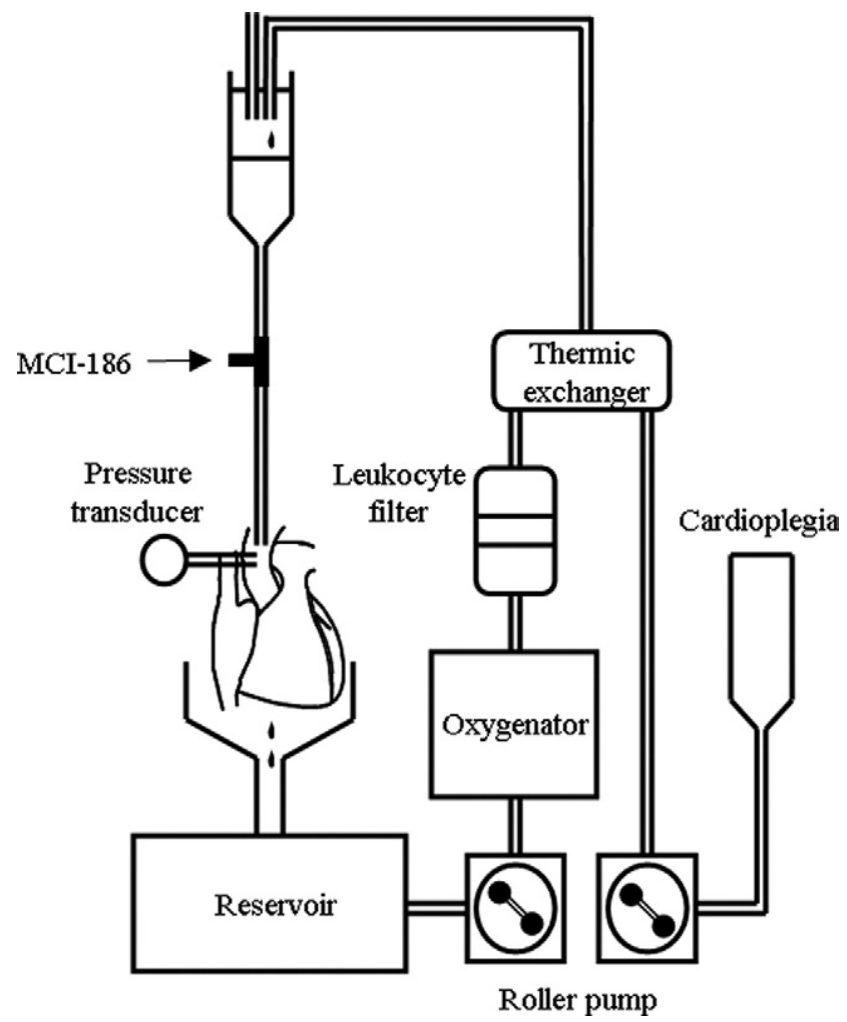

Figure 1. Isolated myocardial perfusion. Blood cardioplegia and blood were infused into coronary arteries through aortic root cannula. $\mathrm{MCl}-186$ was directly infused into coronary artery by isolated myocardial perfusion.

cheal tube was inserted through a tracheostomy, and mechanical ventilation was begun with a tidal volume of $10 \mathrm{~mL} / \mathrm{kg}$.

After a median sternotomy, heparin $(500 \mathrm{U} / \mathrm{kg}$ ) was given intravenously. Catheters were inserted into the carotid artery, the internal jugular vein, and the left atrium for pressure monitoring. A $5 \mathrm{~F}$ thermodilution catheter was inserted into the pulmonary artery trunk for measurement of cardiac output. A 3F pressure-tip catheter (Millar Instruments, Inc, Houston, Tex) and a conductance catheter (2S-RH6DA-116; Alpha Medical Instruments, Mission Viejo, Calif) were inserted from the apex into the left ventricle (LV). A 3F catheter was placed in the coronary sinus to obtain blood samples.

\section{Experimental Protocol}

Asphyxiation was induced by turning the ventilator off. After cardiac arrest was achieved with standstill or ventricular fibrillation, the animals were left for 30 minutes at room temperature. The heart, including both atria, ventricles, and the aortic arch, was excised and weighed. After insertion of a perfusion cannula into the aortic root, the ascending aorta was clamped, and then isolated continuous myocardial perfusion was performed by cardiopulmonary bypass $(\mathrm{CPB})$ with blood cardioplegic solution at $20^{\circ} \mathrm{C}$ with a perfusion pressure of $40 \mathrm{~mm} \mathrm{Hg}$ for 20 minutes (Figure 1). This solution was composed of leukocyte-depleted normoxemic $\left(\mathrm{PO}_{2}\right.$ 
about $100 \mathrm{~mm} \mathrm{Hg}$ ) oxygenated blood mixed with a modified $\mathrm{St}$ Thomas' Hospital solution (20\% D-mannitol $100 \mathrm{~mL}$, sodium bicarbonate $19 \mathrm{mEq}$, potassium chloride $18.5 \mathrm{mEq}$, magnesium sulfate $37 \mathrm{mEq}$, in $1 \mathrm{~L} 5 \%$ glucose) in a 4:1 ratio. Myocardial perfusion was then switched with leukocyte-depleted hyperoxemic oxygenated blood $\left(\mathrm{PO}_{2}\right.$ about $\left.300 \mathrm{~mm} \mathrm{Hg}\right)$ at $20^{\circ} \mathrm{C}$ with a perfusion pressure of $40 \mathrm{~mm} \mathrm{Hg}$. After 20 minutes, the perfusion pressure was elevated to $60 \mathrm{~mm} \mathrm{Hg}$, and the temperature was increased stepwise to $37^{\circ} \mathrm{C}$ during a period of 30 minutes.

In the treated group $(n=6)$, administration of MCI-186 (3 $\mathrm{mg} / \mathrm{kg}$ ) was begun at the time of reperfusion and continued for 30 minutes (until 10 minutes after the initiation of blood reperfusion). The coronary effluent was discarded to keep MCI-186 concentration at a constant level in the circuit. We selected the most effective doses and infusion period of MCI-186 on the basis of well-established dose-response studies of this agent in reperfusion injury. ${ }^{13,14}$ The remaining 6 pigs did not receive MCI-186 at any time during the experiment and served as a control group.

Another $\mathrm{CPB}$ circuit incorporating a pediatric oxygenator (D902 Lilliput 2; Dideco SpA, Mirandola, Italy), filled with $500 \mathrm{~mL}$ donated blood, 10,000 units heparin, and $500 \mathrm{mg}$ methylprednisolone, was established by bicaval and aortic cannulation in a recipient animal and was maintained with a flow rate of $75 \mathrm{~mL} /(\mathrm{kg} \cdot \min )$. At this stage, orthotopic heart transplantation was performed with the donor heartbeat being maintained by continuous myocardial perfusion. Two hours after aortic unclamping, animals were weaned from CPB with an infusion of $0.1 \mu \mathrm{g} /(\mathrm{kg} \cdot \mathrm{min})$ epinephrine. At the end of each experiment, the animals were killed by intravenous injection of potassium chloride.

\section{Cardiac Function}

Cardiac function was assessed before asphyxiation for baseline values. Because respiratory function after transplantation deteriorated with time as result of LV dysfunction, measurements were repeated 1 hour after weaning from CPB. Cardiac output was measured by the thermodilution method. LV end-systolic pressurevolume ratio was calculated from pressure-volume relation curves during transit volume unloading induced by the occlusion of both venae cavae. Maximum and minimum of first derivative of LV pressure were obtained from a pressure curve acquired with a high-fidelity micromanometer catheter. Calibration for blood conductivity was performed just before each measurement.

\section{Determination of Malondialdehyde and Myocardial Fraction of Creatine Kinase}

Blood samples were collected from the aorta and the coronary effluent before anoxia (baseline value), at 5 minutes and 10 minutes during the controlled initial reperfusion with blood cardioplegic solution, and subsequently at 5, 10, 20, 30, 60, 120, and 180 minutes during the perfusion with oxygenated blood. The plasma concentration of malondialdehyde (MDA) was determined with a commercially available colorimetric assay kit (MDA-586 assay kit; OXIS International Inc, Foster City, Calif). The difference in MDA level between the coronary sinus and the aortic root $\left(\mathrm{MDA}_{\Delta}\right)$ was calculated. The myocardial fraction of creatine kinase was determined by a chemiluminescence immunoassay and expressed as a percentage of total creatine kinase.
TABLE 1. Procedure variables

\begin{tabular}{lrr}
\hline & Control group & Treated group \\
\hline Agonal period (min) & $9 \pm 1$ & $9 \pm 1$ \\
Warm ischemic time (min) & $36 \pm 1$ & $37 \pm 1$ \\
Implantation time (min) & $72 \pm 7$ & $73 \pm 5$ \\
Cardiopulmonary bypass time (min) & $218 \pm 6$ & $217 \pm 7$ \\
\hline
\end{tabular}

All values expressed as mean \pm SD. No differences between groups were significant. The agonal period was the time from initiation of asphyxiation to cardiac arrest; the warm ischemic time was the time from cardiac arrest to the beginning of initial controlled reperfusion; the implantation time was the time taken for anastomoses.

\section{Myocardial Edema}

The wet weight of the heart including the aortic arch was measured at the time of excision of the donor heart and at the end of the experiment. The posterior wall thickness of the LV was measured by surface echocardiography with short-axis imaging at the level of papillary muscles before asphyxiation and after transplantation.

\section{Histopathologic Examination}

Biopsy specimens were taken from the LV anterior wall of the beating heart after assessment of posttransplantation cardiac function. For light microscopic examination, the specimens were fixed in $10 \%$ buffered formaldehyde, embedded in paraffin, cut into $5-\mu \mathrm{m}$ thick sections, and stained with hematoxylin and eosin. For transmission electron microscopic study, specimens were fixed in phosphate-buffered ( $\mathrm{pH} 7.4)$ glutaraldehyde (1\%) and paraformaldehyde $(2 \%)$, postfixed in osmium tetroxide $(2 \%)$, and embedded in epoxy. Ultrathin sections were stained with uranyl acetate and lead citrate. A Hitachi H7100 electron microscope (Hitachi, Ltd, Tokyo, Japan) was used to study the sections. Morphologic evaluation was performed by a blinded observer (C.Y.).

\section{Statistical Analysis}

The Wilcoxon signed rank test was used for comparison between baseline and posttransplantation values. Statistical significance of any difference between the groups was determined with the MannWhitney test. Analysis of variance was used to compare MDA . Results are given as mean \pm SD unless otherwise indicated.

\section{Results}

There were no significant differences between the groups in the agonal period (time from the initiation of asphyxiation to cardiac arrest), warm ischemic time, implantation time, and CPB time (Table 1). Baseline values of each variable of cardiac function measured before asphyxiation did not differ between the groups. Flow rate during the initial 20minute reperfusion with blood cardioplegia increased from $50 \mathrm{~mL} / \mathrm{min}$ to $65 \mathrm{~mL} / \mathrm{min}$, indicating reduction in coronary resistance. All hearts started beating slowly approximately 5 minutes after blood reperfusion, spontaneously recovered regular rhythm, and were successfully weaned from CPB. Heart rate, mean pulmonary arterial pressure, left atrial pressure, and central venous pressure after transplantation were significantly higher than baseline values but did not 
TABLE 2. Hemodynamic variables

\begin{tabular}{|c|c|c|c|c|c|c|}
\hline & \multicolumn{3}{|c|}{ Control group } & \multicolumn{3}{|c|}{ Treated group } \\
\hline & BT & PT after CPB & PT $1 \mathrm{~h}$ after CPB & BT & PT after CPB & PT $1 \mathrm{~h}$ after CPB \\
\hline Heart rate (beats/min) & $92 \pm 11$ & $123 \pm 10^{*}$ & $128 \pm 14^{*}$ & $91 \pm 10$ & $120 \pm 13^{*}$ & $125 \pm 8^{*}$ \\
\hline Mean arterial pressure $(\mathrm{mm} \mathrm{Hg})$ & $60 \pm 7$ & $47 \pm 5^{*}$ & $50 \pm 10^{*}$ & $59 \pm 12$ & $48 \pm 3^{*}$ & $53 \pm 5^{*}$ \\
\hline Mean pulmonary arterial pressure $(\mathrm{mm} \mathrm{Hg})$ & $12 \pm 2$ & $25 \pm 3^{*}$ & $26 \pm 7^{*}$ & $13 \pm 3$ & $22 \pm 5^{*}$ & $23 \pm 5^{*}$ \\
\hline Left atrial pressure $(\mathrm{mm} \mathrm{Hg})$ & $3 \pm 2$ & $6 \pm 2^{*}$ & $6 \pm 2^{*}$ & $2 \pm 2$ & $6 \pm 2^{*}$ & $6 \pm 2^{*}$ \\
\hline Central venous pressure $(\mathrm{mm} \mathrm{Hg})$ & $1 \pm 1$ & $5 \pm 2^{*}$ & $7 \pm 3^{*}$ & $1 \pm 1$ & $5 \pm 2^{*}$ & $6 \pm 2^{*}$ \\
\hline
\end{tabular}

$B T$, Before transplantation; $P T$, posttransplantation; $C P B$, cardiopulmonary bypass. All values expressed as mean $\pm S D$. ${ }^{*} P<.05$ versus value before transplantation.

change until 1 hour after CPB (Table 2). The posttransplantation recovery rates of cardiac output, LV end-systolic pressure-volume ratio, and maximum and minimum of first derivative of $\mathrm{LV}$ pressure in the treated group remained at approximately $80 \%$ of baseline values but were all significantly better than those in the control group (Table 3).

The changes in $\mathrm{MDA}_{\Delta}$ during reperfusion are shown in Figure $2 . \mathrm{MDA}_{\Delta}$ in the treated group remained low throughout the reperfusion. On the other hand, $\mathrm{MDA}_{\Delta}$ in the control group abruptly increased after initiation of oxygenated blood perfusion, although values were low during the initial reperfusion with blood cardioplegia. Release of the myocardial fraction of creatine kinase, an index of myocyte injury, increased after transplantation in both groups, but the mean enzyme level in the treated group was significantly lower than that in the control group $(323 \pm 56 \mathrm{IU} / \mathrm{L}$ vs $741 \pm 161 \mathrm{IU} / \mathrm{L}, P<.05)$.

Although heart weight increased after transplantation in both groups, weight gain in the treated group was significantly less than in the control group $(6 \% \pm 2 \%$ vs $11 \% \pm$ $1 \%, P<.05)$. LV posterior wall thickness increased after transplantation in all hearts, but the mean thickening of the treated hearts was significantly smaller than that of the untreated hearts $(11 \% \pm 3 \%$ vs $20 \% \pm 4 \%, P<.05)$.

Hematoxylin-eosin staining demonstrated smaller eosinophilic myocardial changes, less edema, and less vacuolar degeneration in the treated group than in the control group, although fundamental myocardial structures were preserved in both groups (Figure 3). Transmission electron micrographs demonstrated that the myocardium of the untreated hearts had marked swelling of mitochondria, with disruption of the cristae, dilated endoplasmic reticulum, and scant glycogen granules. In contrast, the treated hearts showed well-preserved ultrastructure, including mitochondria with regular cristae. Other organelles, such as endoplasmic reticulum, Golgi apparatus, T-tubules, and so on were of normal appearance (Figure 4). These findings indicated that there was no irreversible ischemic change in the treated hearts.

\section{Discussion}

Although a number of approaches to procurement of cadaver hearts have been studied in the laboratory, understanding of the outcomes is confounded by several experimental factors. Martin and colleagues ${ }^{15}$ showed well-preserved myocardial viability 24 hours after transplantation from exsanguinated NHBDs by refined preservation techniques but without pretreatment. There is some evidence, however, that death from asphyxiation in a possible cardiac allograft NHBD causes more severe myocardial damage than does death from exsanguination. ${ }^{16}$ Regarding postmortem ischemic time, a Pittsburgh group ${ }^{17}$ reported that in the patients undergoing liver and kidney transplantation from NHBDs whose families had requested that life support be withdrawn (category 3, The Maastricht International Workshop ${ }^{18}$ ), the mean interval between cardiac arrest and thoracic aorta crossclamping was 24 minutes. We used pigs in this study because of similarities of the myocardium and the coronary anatomy to those of human beings. To mimic the category 3 NHBD situation, we used the asphyxiation model and set the duration of postmortem ischemia at 30 minutes.

TABLE 3. Recovery rate of cardiac function

\begin{tabular}{|c|c|c|c|c|c|c|}
\hline & \multicolumn{3}{|c|}{ Control group } & \multicolumn{3}{|c|}{ Treated group } \\
\hline & & & Recovery rate & & & Recovery rate \\
\hline & BT & PT & $(\%)$ & BT & PT & $(\%)$ \\
\hline Cardiac output (L/min) & $1.9 \pm 0.3$ & $1.2 \pm 0.1$ & $60 \pm 10$ & $2.0 \pm 0.2$ & $1.6 \pm 0.1$ & $81 \pm 5^{*}$ \\
\hline LV end-systolic pressure-volume ratio (mm Hg/mL) & $4.8 \pm 0.3$ & $3.0 \pm 0.3$ & $62 \pm 7$ & $4.8 \pm 0.5$ & $4.0 \pm 0.3$ & $82 \pm 4^{*}$ \\
\hline Maximum LV developed pressure (mm Hg/s) & $1180 \pm 280$ & $723 \pm 105$ & $63 \pm 8$ & $1219 \pm 249$ & $1024 \pm 101$ & $86 \pm 10^{*}$ \\
\hline Minimum LV developed pressure (mm Hg/s) & $829 \pm 204$ & $524 \pm 116$ & $64 \pm 8$ & $824 \pm 115$ & $726 \pm 62$ & $89 \pm 8^{*}$ \\
\hline
\end{tabular}

$B T$, Before transplantation; $P T$, posttransplantation; $L V$, left ventricular. All values expressed as mean $\pm \mathrm{SD}$. ${ }^{*} P<.05$ versus control group. 


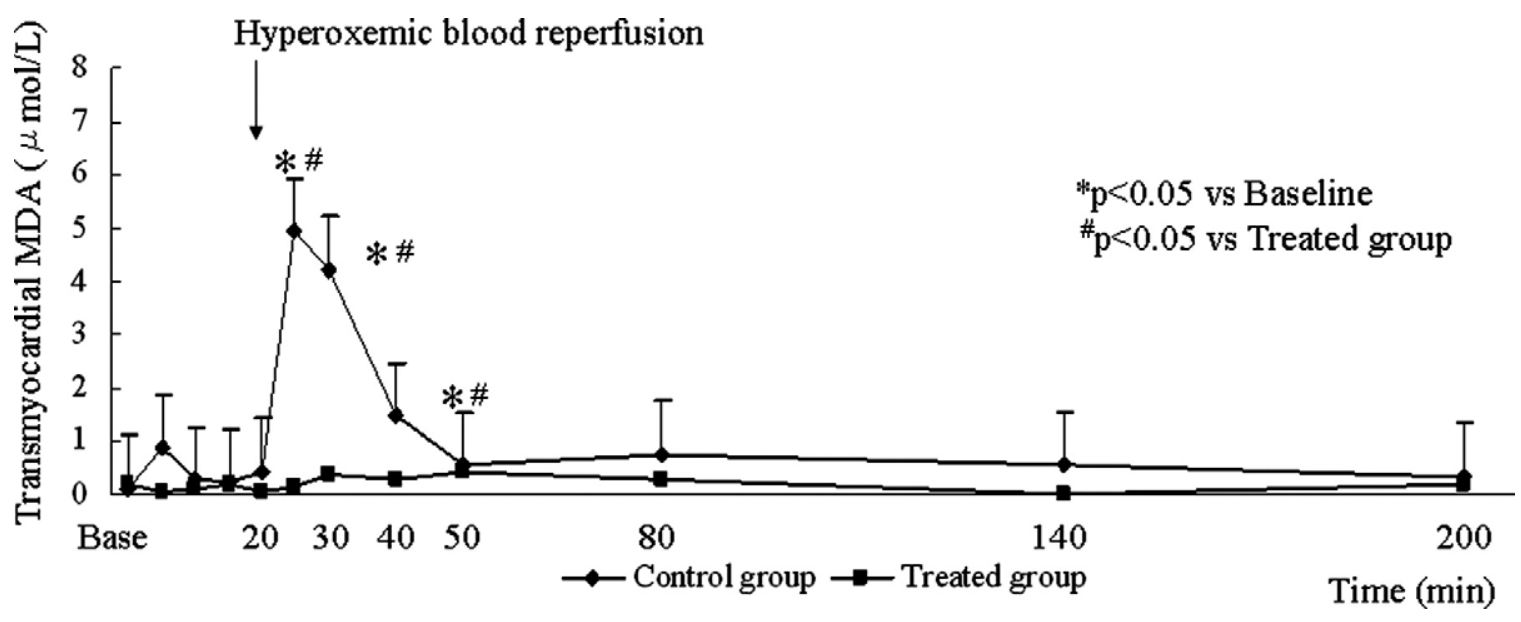

Figure 2. Changes in coronary difference in levels of malondialdehyde (MDA) between sinus and aortic root. Values are mean \pm SD.

Resuscitation of nonbeating hearts by early resumption of myocardial perfusion is beneficial in reducing ischemia. Deleterious events initiated by reperfusion, however, such as myocardial hypercontracture, cytotoxic reactive oxygen species, complement activation, and neutrophil adhesion, can result in irreversible tissue injury. We recently reported that controlled initial reperfusion with leukocyte-depleted lukewarm blood cardioplegia at $40 \mathrm{~mm} \mathrm{Hg}$ for 20 minutes provided better posttransplantation graft function than if the initial reperfusion lasted for 5 or 60 minutes, although the recovery rate was at best $60 \% .^{6}$ Results of this study demonstrate that supplementation of the reperfusate with MCI186 reduced leakage of the myocardial fraction of creatine kinase, prevented myocardial edema, preserved better myocardial structure, and enhanced functional recovery, indicating that antioxidant therapy at the initiation of reperfusion is essential to successful resuscitation of nonbeating hearts.

The main sources of free-radical production in myocardial tissue in ischemia-reperfusion are thought to involve the xanthine oxidase-hypoxanthine system, the electron transmission system in mitochondria, and the arachidonic cascade and the reduced nicotinamide adenosine diphosphate oxidase system in polymorphonuclear white blood cells. Although superoxide generated as the initial radical through these systems is less toxic, the most potent oxidizer, the hydroxyl radical, can be formed from superoxide by an iron-catalyzed Haber-Weiss reaction. ${ }^{7}$ Hydroxyl radical accelerates lipid peroxidation of cell membranes, which causes increased permeability of membrane structures of all the main organelles to calcium ion, resulting in hypercontracture of myofibrils. ${ }^{8}$ In addition, the metabolites of arachidonic acid, which are produced through the lipoxygenase pathways, exert deleterious cardiovascular effects. Leukotrienes, hydroxyeicosatraenoic acids, and hydroxyeicosatet- raenoic acids induce vasoconstriction, particularly constriction of the coronary vascular beds. Japanese investigators have reported that MCI-186 scavenges hydroxyl radicals, inhibits the lipoxygenase pathway in the arachidonic acid cascade, ${ }^{19,20}$ and prevents both hydroxyl radical-dependent and iron-dependent lipid peroxidation of the cell membrane. ${ }^{10,11}$

MDA originates from an oxygen free radical-mediated breakdown of peroxidized phospholipids. ${ }^{21}$ In this study, enhancement of posttransplantation functional recovery in MCI-186-treated hearts was associated with marked suppression of MDA production, suggesting that this compound exerts its cardioprotective property through inhibition of detrimental lipid peroxidation. Although $\mathrm{MDA}_{\Delta}$ during the controlled initial reperfusion with normoxemic blood cardioplegia remained at negligible levels in both groups, values in the untreated group increased after conversion of the perfusion solution to hyperoxemic blood. Ihnken and associates ${ }^{22}$ found in their hypoxemic piglet models that acute reoxygenation $(400 \mathrm{~mm} \mathrm{Hg}$ ) by $\mathrm{CPB}$ reduced the antioxidant reserve capacity and that normoxemic blood cardioplegia further suppressed lipid peroxidation and better preserved cardiac contractility than did hyperoxemic blood cardioplegia. Taking these findings with our results, we suspect that normoxemic blood cardioplegia itself may have antioxidant effects and that acute reoxygenation, which can lead to a burst of free-radical generation, should be avoided during continuous myocardial perfusion in nonbeating hearts.

To determine the optimal timing and duration of administration of antioxidant agents, it is important to identify the exact time window during which free radical-mediated myocardial damage occurs. Previous studies demonstrated that free-radical scavengers exert cardioprotective properties when they are administered just before or at the time 

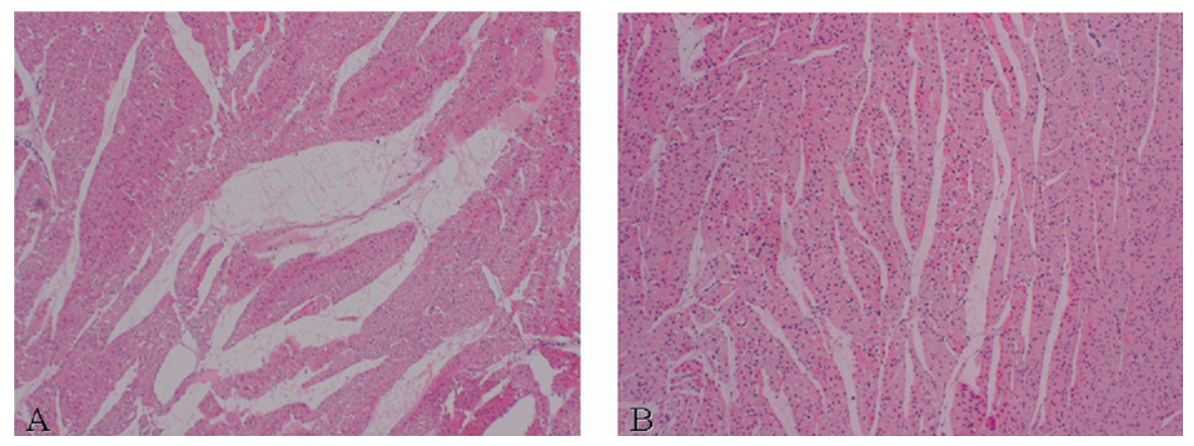

Figure 3. Light micrographs of untreated myocardium (A) and $\mathrm{MCl}-186-$ treated myocardium (B) after transplantation (hematoxylin-eosin, $\times 40$ ).

of reperfusion after total or regional global ischemia, ${ }^{23}$ whereas no such effect manifests when they are given 1 minute after initiation of reperfusion. Although we administered MCI-186 during the first 30 minutes of reperfusion, from beginning of the controlled initial reperfusion with normoxemic blood cardioplegia until 10 minutes after hyperoxemic blood perfusion, the antioxidant effect of MCI186 assessed by MDA production was noticed immediately after conversion of perfusion solution to hyperoxemic blood. Because free-radical generation and subsequent tissue injury are proportionate to $\mathrm{PaO}_{2},{ }^{24,25}$ gradual reoxygenation during reperfusion could avoid a burst of free radicals in globally ischemic hearts.

Myocardial edema is associated with cardiac dysfunction; as edema accumulates within the interstitial spaces, interstitial pressure rises, thus increasing the stiffness and decreasing the compliance of both ventricles. ${ }^{26,27}$ Blood cardioplegia itself causes edema through the combination of increased myocardial fluid filtration and decreased cardiac lymph drainage. ${ }^{28}$ The hearts in both groups increased in wall thickness and weight and showed significant edema after transplantation, but the MCI-treated hearts had less edema than did the untreated hearts. Our results indicate that MCI-186 exerts its cardioprotective effects at least in part by preventing myocardial edema.

For the last three decades, effects of global ischemia on the myocardium have been extensively investigated in experimental animal models, but conclusions with regard to the degree of myocardial injury and its reversibility have been controversial. Schaper and coworkers ${ }^{29}$ studied the effects of various durations of global ischemia and reperfusion in the myocardium of isolated dog hearts and showed that although functional, biochemical, and ultrastructural changes tended to recover during the reperfusion phase, even in severely injured hearts except "stone heart," the reconstitution of the myocardial ultrastructure occurred at a faster rate than did recovery of other parameters. Subcellular structural integrity thus may be a prerequisite for reversible ischemic injury. Because the MCI-treated hearts had well-preserved ultrastructure, further improvement of cardiac function can be expected by longer support with continuous myocardial perfusion before transplantation.

In conclusion, without the use of any cardioprotective pretreatment, resuscitation of nonbeating pig hearts obtained after 30 minutes of global ischemia is feasible by means of continuous myocardial perfusion, initially with leukocyte-depleted lukewarm-blood cardioplegia for 20 minutes and then with oxygenated blood. A free-radical scavenger, MCI-186, when administered for the first 30 minutes of reperfusion provides better preservation of myocardial structure and enhanced recovery of cardiac function after transplantation, at least in part by inhibition of lipid peroxidation. Before NHBDs are used in human heart transplantation, modalities to determine transplantability must be established. At this time, electron microscopy of small tissue samples obtained during resuscitation appears to be
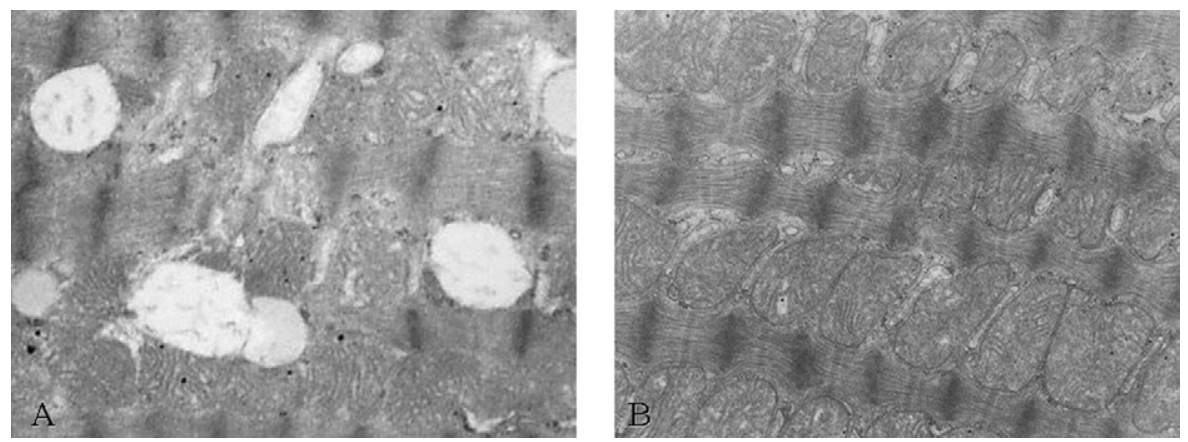

Figure 4. Transmission electron micrographs of untreated myocytes $(A)$ and MCl-186-treated myocytes (B) after transplantation (hematoxylin-eosin, $\times 10000$ ). 
the only clinically applicable method to estimate the reversibility of the ischemic state of the heart. ${ }^{29}$

We thank Ms. Masumi Furutani (Central Research Laboratory, Okayama University Graduate School of Medicine and Dentistry), Akiko Itagaki, and Saori Tsukuda for technical assistance.

\section{References}

1. Shirakura R, Matsuda H, Nakano S, Nakata S, Kaneko M, Miyamoto $\mathrm{Y}$, et al. Cardiac function and myocardial performance of 24-hourpreserved asphyxiated canine hearts. Ann Thorac Surg. 1992;53:440-4.

2. Gundry SR, Fukushima N, Eke CC, Hill AC, Zuppan C, Bailey LL. Successful survival of primates receiving transplantation with "dead," nonbeating donor hearts. J Thorac Cardiovasc Surg. 1995;109:1097102.

3. Wuerflein R, Shumway N. Resuscitation and function of the cadaver heart. Circulation. 1966;35(4 Suppl):I92-5.

4. Tam W, Robicsek F, Daugherty HK. The autoperfusing heart-lung preparation: a vehicle for the preservation of the resuscitated cadaver heart. J Thorac Cardiovasc Surg. 1969;58:879-85.

5. Hearse DJ, Humphrey SM, Chain EB. Abrupt reoxygenation of the anoxic potassium-arrested perfused rat heart: a study of myocardial enzyme release. J Mol Cell Cardiol. 1973;5:395-407.

6. Osaki S, Ishino K, Kotani Y, Honjo O, Suezawa T, Kanki K, et al. Resuscitation of non-beating donor hearts using continuous myocardial perfusion: the importance of controlled initial reperfusion. Ann Thorac Surg. 2006;81:2167-71.

7. McCord JM. Oxygen-derived free radicals in postischemic injury. N Engl J Med. 1985;312:159-63.

8. Meerson FZ, Kagan VE, Kozlov YP, Belkina LM, Arkkhipenko YV. The role of lipid peroxidation in pathogenesis of ischemic damage and the antioxidant protection of the heart. Basic Res Cardiol. 1982;77: 465-85.

9. Mizuno A, Uemura K, Nakashima M. Inhibitory effect of MCI-186, a free radical scavenger, on cerebral ischemia following rat middle cerebral artery occlusion. Gen Pharmacol. 1998;30:575-8.

10. Watanabe T, Yuki S, Egawa M, Nishi H. Protective effects of MCI186 on cerebral ischemia: possible involvement of free radical scavenging and antioxidant actions. J Pharmacol Exp Ther. 1994;268: 1597-604.

11. Yagi H, Horinaka S, Matsuoka H. Edaravone prevented deteriorated cardiac function after myocardial ischemia-reperfusion via inhibiting lipid peroxidation in rat. J Cardiovasc Pharmacol. 2005;46:46-51.

12. Yoneda Y, Uehara T, Yamasaki H, Kita Y, Tabuchi M, Mori E. Hospital-based study of the care and cost of acute ischemic stroke in Japan. Stroke. 2003;34:718-24.

13. Yanagisawa A, Miyagawa M, Ishikawa K, Murota S. Cardioprotective effect of MCI-186 (3-methyl-1-phenyl-2-pyrazolin-5-one) during acute ischemia-reperfusion injury in rats. Int J Angiol. 1994;3:12-5.
14. Takamatsu Y, Yuki S, Watanabe T. [Studies on the concentrations of 3-methyl-1-phenyl-2-pyrazolin-5-one (MCI-186) in MCA occlusion and reperfusion model of rats.] Jpn Pharmacol Ther. 1997;25 Suppl: S1785-91. Japanese.

15. Martin J, Lutter G, Ihling C, Siepe M, Wagner S, Hilberath J, et al. Myocardial viability twenty-four hours after orthotopic heart transplantation from non-heart beating donors. J Thorac Cardiovasc Surg. 2003; 125:1217-28.

16. Lundsgaard-Hansen P, Schilt W, Heitmann L, Oroz M, Buchler A, Lemeunier A. Influence of the agonal period on the postmortem metabolic state of the heart. A problem in cardiac preservation. Ann Surg. 1971;174:744-54.

17. Casavilla A, Ramirez C, Shapiro R, Nghiem D, Miracle K, Bronsther $\mathrm{O}$, et al. Experience with liver and kidney allografts from non-heart beating donors. Transplantation. 1995;59:197-203.

18. Kootsta G, Daemen JH, Oomen AP. Categories of non-heart beating donors. Transplant Proc. 1995;27:2893-4.

19. Watanabe T, Morita I, Nishi H, Murota S. Preventive effect of MCI186 on 15-HPETE induced vascular endothelial cell injury in vitro. Prostaglandins Leukot Essent Fatty Acids. 1988;33:81-7.

20. Watanabe T, Egawa M. Effects of an antistroke agent MCI-186 on cerebral arachidonate cascade. J Pharmacol Exp Ther. 1994;271: 1624-9.

21. Di Pierro D, Tavazzi B, Lazzarino G, Giardina B. Malondialdehyde is a biochemical marker of peroxidative damage in the isolated reperfused rat heart. Mol Cell Biochem. 1992;116:193-6.

22. Ihnken K, Morita K, Buckberg GD, Sherman MP, Ignarro LJ, Young HH. Studies of hypoxemic/reoxygenation injury: with aortic clamping. $J$ Thorac Cardiovasc Surg. 1995;110:1274-86.

23. Ambrosio G, Weisfeldt ML, Jacobus WE, Flaherty JT. Evidence for a reversible oxygen radical-mediated component of reperfusion injury: reduction by recombinant human superoxide dismutase administered at the time of reflow. Circulation. 1987;75:282-91.

24. Morita K, Ihnken K, Buckberg GD. Role of controlled cardiac reoxygenation in reducing nitric oxide production and cardiac oxidant damage in cyanotic infantile hearts. J Clin Invest. 1994;93:2658-66.

25. Littauer A, DeGroot H. Release of reactive oxygen by hepatocytes on reoxygenation: three phases and role of mitochondria. Am J Physiol. 1992;262:G1015-20.

26. Laine GA, Allen SJ. Left ventricular myocardial edema: lymph flow, interstitial fibrosis, and cardiac function. Circ Res. 1991;68:1713-21.

27. Garcia-Dorado D, Oliveras J. Myocardial edema: a preventable cause of reperfusion injury? Cardiovasc Res. 1993;27:1555-63.

28. Mehlhorn U, Allen SJ, Adams DL, Davis KL, Gogola GR, de Vivie ER, et al. Normothermic continuous antegrade blood cardioplegia does not prevent myocardial edema and cardiac dysfunction. Circulation. 1995;92:1940-6.

29. Schaper J, Mulch J, Winkler B, Schaper W. Ultrastructural, functional, and biochemical criteria for estimation of reversibility of ischemic injury: a study on the effects of global ischemia on the isolated dog heart. J Mol Cell Cardiol. 1979;11:521-41. 